OPEN ACCESS

Edited by:

Qinghua He,

Southwest University,

China

Reviewed by:

Xiongying Chen,

Capital Medical University,

China

Leilei Mei,

South China Normal University,

China

*Correspondence:

Yun Wang

wangyun@bnu.edu.cn

Specialty section:

This article was submitted to

Psychopathology,

a section of the journal

Frontiers in Psychiatry

Received: 29 March 2019

Accepted: 21 May 2019

Published: 13 June 2019

Citation:

Ke L, Duan W, Xue Y and Wang Y (2019) Developmental Coordination

Disorder in Chinese Children is Correlated With Cognitive Deficits.

Front. Psychiatry 10:404.

doi: 10.3389/fpsyt.2019.00404

\section{Developmental Coordination Disorder in Chinese Children Is Correlated With Cognitive Deficits}

\author{
Li Ke ${ }^{1}$, Wen Duan ${ }^{2}$, Ye Xue ${ }^{1}$ and Yun Wang ${ }^{1 *}$ \\ ${ }^{1}$ State Key Laboratory of Cognitive Neuroscience and Learning, Beijing Normal University, Beijing, China, ${ }^{2}$ Collaborative \\ Innovation Center of Assessment for Basic Education Quality, Beijing, China
}

Cognitive deficits have been commonly observed in children with developmental coordination disorder (DCD), including memory, attention, and executive function difficulties. The present study evaluates the specific cognitive deficits in Chinese children with DCD, through a number of tests. A total of 401 children aged 7 to 10 years old from primary schools in Guangdong Province, China, participated in this study. Using the guidelines of the Movement Assessment Battery for Children ("Movement ABC-2"), a measurement tool of motor function ability, the children were divided into a DCD group, a group identified as being at risk of DCD, and a normal control group. The results of our analysis revealed that children's overall motor abilities could predict their overall cognitive ability, reaction time, memory, and attention. The performance of the DCD children was worse than that of the other two groups in terms of reaction time. The DCD group also returned lower scores for executive function than the normal control group did. A regression analysis showed that the cognitive deficits in children with DCD center mainly on poor executive function rather than attention and memory issues. These findings provide preliminary results regarding the cognitive deficits in Chinese children with DCD and have potential applications for the diagnosis and treatment of the disorder.

Keywords: developmental coordination disorder, movement development, cognitive ability, cognitive deficits, execution function

\section{INTRODUCTION}

Movement development corresponds to other basic abilities and has attracted much attention from researchers as well as health-care providers. Previous studies have labeled children's movement difficulties as clumsiness, dyspraxia, motor learning difficulties, or as a disorder of attention, motor control, and perception ("DAMP"). These concepts explain children's problems with movement or the acquisition of movement from different perspectives (1-5). However, such terminologies only focus on a particular field or the children's symptoms but not their developmental process (6). Through in-depth explorations, researchers have ultimately reached a consensus that the developmental coordination disorder (DCD) of children should be classified as a subcategory of a neurodevelopmental disorder $(7,8)$.

Currently, DCD is generally identified as an impairment of motor ability distinct from intelligence and other neurological disorders (8). With the development of brain and cognitive neuroscience research, though, it has been discovered that DCD is not just relevant to the movement system, but is also related to a complex series of neurological dysfunctions involving memory, attention, executive 
function, and reasoning $(9,10)$, each of which is discussed further in the paragraphs that follow. Additionally, neuroimaging data has shown altered brain activation patterns across functional networks involving prefrontal, parietal, and cerebellar regions in children with DCD, compared to children in other groups (11-13), which overlap with a couple of key structures within the internal modeling regions (14). Moreover, researchers have found evidence that is suggestive of a relationship between DCD and profound cognitive deficits. Research in overweight 7-11 year-old children found that exercise improved executive function and altered brain activation (15).

Children with DCD often suffer a loss of visual-spatial memory, which is significantly correlated with the planning and control of their movements $(16,17)$. This may be due to a different number of cognitive resources being allocated to visual-spatial memory during the process of executing actions (18). Furthermore, a close relationship between visual-spatial memory and learning ability among children with DCD (19) has been found, with research showing that, among children with DCD, those with lower visual-spatial memory perform less well in tests on their literacy and computation abilities (20). The particular correlation between visual-spatial memory and learning ability indicates that the processing of working memory and visual-spatial memory plays an important role in the acquisition process of movement development for children with DCD.

Furthermore, children with DCD also exhibit poor performances in attentional tasks that require autonomous processing of visual-spatial information, such as cue information (21), visual tracking (22), and simultaneous responses to dual tasks (23). Prior research featured direction-hiding visual-spatial attention tasks to verify the relationship between DCD and a lack of selective attention, and found that children with DCD experienced longer reaction times in the presence of external disturbances, in comparison with children in the normal control group $(24,25)$. Subsequently, Wilmut et al. (26) found that the slow response to exogenous attention distribution among children with DCD was only observed in tasks involving the integration of visual actions. To further clarify the relationship between attention and movement performance among children with DCD, more recent studies have used electroencephalogram recordings taken from the foreheads of participants and subsequently converting them into indicators of attentional levels, with the results indicating a correlation between attention level and movement performance among children with DCD (27).

"Reaction time" represents an individual's fastest response to the occurrence of a stimulus. Researchers have found that the cognitive decision-making processes associated with responding to choices results in the relatively slower movements of children with DCD; that is, they have longer reaction times than other children $(21,28-30)$. These findings suggest that children with DCD have difficulties processing movement information.

Dysfunction in execution is also an important characteristic of children with DCD. Indeed, a meta-analysis of research, carried out during the period of 1997 to 2011, and relevant to the assessment of movement planning, inhibition, working memory, and cognitive flexibility indicated significant executive dysfunction (31). "Executive function" is a complex process comprising higher-order cognitive thought and action that involves working memory, inhibition control, and cognitive flexibility (32). Impaired executive function can impact metacognition when making movement plans, which in turn suggests that children with DCD may experience extensive problems with regard to this aspect too.

Our analysis of previous research establishes that cognitive deficits are commonly seen in children with DCD, as deficits can be observed with respect to their memory, attention, and executive function. These prior findings prompted our current research question, as follows: What is the correlation between memory, attention, and executive function among children with DCD-and is there a relationship of inclusion between these factors? As previous studies have mainly featured Western children as participants, we posited a further question of whether similar results could be obtained in China. For the present study, we screened a designated sample size of children for DCD using a standardized tool with a credible norm. We subsequently conducted cognitive tests, involving memory, attention, executive function, and reaction time. The purpose of our research was to verify and analyze the overall impact of DCD on children's cognitive abilities and specific cognitive functions, such as memory, attention, and executive function, as well as to explore cognitive deficits in the dynamic mechanisms underlying children's movement development and difficulties.

\section{MATERIALS AND METHODS}

\section{Participants}

A total of 401 children from three primary schools in Guangdong Province, China, participated in the movement and cognitive tests in this study. The children were aged 7 to 10 years old (mean age $=8.98 \pm 1.10$ years) and the sample consisted of 214 boys and 187 girls. The study protocol was screened and approved by the Institutional Review Board of the National Key Laboratory of Cognitive Neuroscience and Learning, Beijing Normal University. Parents were provided with an explanation of the study's procedures. Written and informed consent were obtained from the parents. After the deletion of missing data, the final sample comprised 399 participants. The gender and age distributions of all tested children are shown in Table 1.

Using the guidelines of the Movement Assessment Battery for Children (second edition, also known as "Movement ABC-2"), a measurement tool of motor function ability, children with a total score of 5 points or fewer were assigned to the DCD group; children with a score of 6 were identified as being "at risk" of DCD, and assigned to the AR group; and children with a score of 7 or more were identified as "normal controls" and placed in the NC group accordingly. Applying this standard, the resultant DCD group comprised 75 participants, the AR group had 35, and the NC group featured 291 participants. 
TABLE 1 | Age and gender distributions of participants.

\begin{tabular}{lccc}
\hline Age & Gender & No. of Children & $\%$ \\
\hline 7 years old & Boys & 37 & 64.9 \\
& Girls & 20 & 35.1 \\
8 years old & Boys & 45 & 60.8 \\
& Girls & 29 & 39.2 \\
9 years old & Boys & 40 & 55.6 \\
\multirow{2}{*}{10 years old } & Girls & 50 & 48.9 \\
& Boys & 92 & 64.9 \\
\hline Total & Girls & 88 & 35.1 \\
\hline
\end{tabular}

\section{Methods}

\section{Movement Assessment Battery for Children}

The children completed the Movement ABC-2's "age band 2" tests for 7- to 10-year-old (6). Each age band encompasses three dimensions: 1) manual dexterity, comprising three items; 2 ) aiming and catching, featuring two items; and 3) balance, with three items. For children aged 7 to 10 years, a "normal" model was provided for the span of every single year. Through comparison with these normal models, a standard conversion was used for the tasks, dimensions, and total scores. The converted standard score was 10 , the standard deviation was 3 , and the scores ranged between 1 and 19 .

In 2016, the Chinese norm for children aged between 3 and 10 years was established using this tool. Through stratified sampling, all of China was divided into seven regions: North China, East China, South China, Central China, Southwest China, Northeast China, and Northwest China. Based on the proportion of children under 10 years old in the 2010 census data, the regional sample size was distributed, and data collected from 2,185 children, incorporating 1,143 boys $(52.3 \%)$ and 1,042 girls (47.7\%), showing reasonable gender distributions at all ages. Following data cleaning, analysis, and standard demarcation, the converted standard scores of the Chinese norm were obtained. The test-retest reliability of this Chinese norm is 0.86 and 0.859 in intraclass correlations. The results of confirmatory factor analyses show that the comparative fit index is 0.937 , the root mean square error of approximation is 0.045 , and the TuckerLewis index is 0.897 , signifying good construct validity (33).

\section{Measurement of Cognitive Abilities}

Four core abilities were measured: 1) attention, 2) memory, 3) reaction time, and 4) execution function. Selective attention and continuous attention tasks were used to measure attention. Associative memory and short-term memory tasks were chosen to evaluate memory. Processing speed tasks were used to test reaction time, and impulse control and information-updating tasks were used to measure execution function. The children were asked to complete all cognitive tasks independently on a computer within $30 \mathrm{~min}$. All scores were added, and the total scores were transformed into a standard score with a mean of 100 and a standard deviation of 15 .

\section{Family Background Information}

The parents of participants were asked to fill out a questionnaire in order to provide information regarding each child's mother's years of education, father's years of education, and annual household income. These variables were controlled during the data analysis.

\section{Measurement of Movement Development}

Prior to these measurements being taken, an email was sent to the school administrators that included the Institutional Review Board approval letter, the task lists, and informed consent. These were then distributed to the students' parents or caregivers. The online testing system generated lists of identification numbers for each school according to the number of students who volunteered to complete the study (via their parents). The data were collected via a face-to-face test using the Movement ABC-2 measurement tool on all the participating children.

\section{Measurement of Cognitive Ability}

Children completed their cognitive assessment on a school computer. The interval between the measurement of movement development and the measurement of cognitive ability was more than $2 \mathrm{~h}$.

\section{Data Analysis}

SPSS 20.0 was used for the analyses of the Pearson correlations, multiple linear regression, and multivariate covariance analyses.

\section{RESULTS}

\section{Correlations Between Movement Development and Cognitive Abilities}

The correlations between the total scores for the children's movement development (all dimensions) and cognitive abilities were calculated respectively, and the results are presented in Table 2. As can be seen, the total scores of the children's movement development and their total cognitive scores are significantly correlated $(r=0.18, p<0.01)$, and movement development is also highly correlated to reaction time $(r=0.14, p<0.01)$, memory $(r=0.14, p<0.01)$, attention $(r=0.10, p<0.05)$, and executive function $(r=0, p<0.05)$. The manual dexterity dimension of movement development is significantly correlated to memory $(r=0.13, p<0.05)$, executive function $(r=0.11, p<0.05)$, and the total cognitive scores $(r=0.12, p<0.05)$. The aiming and catching dimension are closely related to attention $(r=0.14, p<$ $0.01)$ and total cognitive score $(r=0.14, p<0.01)$. The balance dimension of movement development is largely correlated to reaction time $(r=0.17, p<0.01)$ as well as total cognitive score $(r=0.13, p<0.05)$.

\section{Relationship Between Movement Development and Cognitive Development}

For the multiple linear regression analysis, the total score for movement development was used as the independent variable; the total score for cognitive abilities as the dependent variable; and the children's genders, ages, parental years of education, and annual family income as the control variables. The results are shown in Table 3, where it can be observed that overall motor 
TABLE 2 | Correlations between cognitive development and movement development.

\begin{tabular}{|c|c|c|c|c|c|c|c|c|c|c|}
\hline & & 1 & 2 & 3 & 4 & 5 & 6 & 7 & 8 & 9 \\
\hline 1 & Cognition-reactive time & 1 & & & & & & & & \\
\hline 2 & Cognition-memory & $0.34^{\star \star}$ & 1 & & & & & & & \\
\hline 3 & Cognition-attention & $0.21^{\star *}$ & $0.20^{\star *}$ & 1 & & & & & & \\
\hline 4 & Cognition-executive function & $0.17^{\star \star}$ & $0.12^{\star}$ & $0.33^{\star \star}$ & 1 & & & & & \\
\hline 5 & Total cognitive score & $0.54^{\star *}$ & $0.47^{\star \star}$ & $0.76^{\star \star}$ & $0.59^{\star \star}$ & 1 & & & & \\
\hline 6 & Movement-manual dexterity & 0.08 & $0.13^{\star}$ & 0.05 & $0.11^{*}$ & $0.12^{*}$ & 1 & & & \\
\hline 7 & Movement-aiming and catching & 0.05 & 0.09 & $0.14^{\star \star}$ & 0.07 & $0.14^{\star \star}$ & $0.20^{\star \star}$ & 1 & & \\
\hline 8 & Movement-balance & $0.17^{\star \star}$ & 0.08 & 0.04 & 0.08 & $0.13^{*}$ & $0.37^{\star \star}$ & $0.18^{\star \star}$ & 1 & \\
\hline 9 & Total movement development score & $0.14^{\star \star}$ & $0.14^{\star *}$ & $0.10^{\star}$ & $0.12^{*}$ & $0.18^{\star \star}$ & $0.71^{\star \star}$ & $0.57^{\star \star}$ & $0.82^{* \star}$ & 1 \\
\hline
\end{tabular}

TABLE 3 | Predictive effect of total movement development score on total cognitive score.

\begin{tabular}{lcccc}
\hline & $\boldsymbol{\beta}$ & $\boldsymbol{t}$ & $\boldsymbol{\Delta} \boldsymbol{R}^{\mathbf{2}}$ & $\boldsymbol{\Delta} \boldsymbol{F}$ \\
\hline Gender & -0.09 & -1.56 & & \\
Age & -0.07 & -1.19 & & \\
Mother's years of education & -0.08 & -1.10 & 0.02 & 1.08 \\
Father's years of education & 0.06 & 0.89 & & \\
Annual household income & -0.01 & -0.22 & & \\
\hline Total movement development score & $0.22^{* * *}$ & 3.89 & $0.05^{* * *}$ & 15.11 \\
\hline *** $p<0.001$ & & & &
\end{tabular}

ability has a significant predictive effect on the overall cognitive ability of the children $(\beta=0.22, p<0.001)$.

The individual scores for reaction time, memory, attention, and executive function were then used as dependent variables for further multiple linear regression analysis, with the independent and control variables as above. The results are presented in Tables 4 to 7 , wherein the overall motor abilities of the children show a significant predictive effect on their reactive abilities $(\beta=0.19$, $p<0.001)$, memory $(\beta=0.20, p<0.001)$, and attention $(\beta=0.14$, $p<0.05$ ), whereas it shows little predictive effect on the children's executive function $(\beta=0.10, p>0.05)$.

\section{Cognitive Abilities of Children With DCD}

In order to study the cognitive differences between the DCD group, AR group, and NC group, a multivariate analysis of covariance

TABLE 4 | Predictive effect of total movement development score on reaction time score.

\begin{tabular}{lcccc}
\hline & $\boldsymbol{\beta}$ & $\boldsymbol{t}$ & $\boldsymbol{\Delta} \boldsymbol{R}^{\mathbf{2}}$ & $\boldsymbol{\Delta} \boldsymbol{F}$ \\
\hline Gender & -0.01 & -0.23 & & \\
Age & $0.33^{\star \star \star}$ & 5.98 & & \\
Mother's years of education & -0.09 & -1.32 & $0.11^{\star \star \star}$ & 7.59 \\
Father's years of education & 0.08 & 1.13 & & \\
Annual household income & 0.00 & 0.02 & & \\
\hline Total movement development score & $0.19^{* \star *}$ & 3.42 & $0.03^{\star \star}$ & 11.72 \\
\hline${ }^{* *} p<0.01$ & & & & \\
${ }^{* * *} p<0.001$. & & & &
\end{tabular}

was conducted with the three groups as independent variables; cognitive ability, reactivity, memory, attention, and executive function scores as dependent variables; and the children's genders, ages, parental years of education, and annual family income as covariates. The results are shown in Table 8.

Here, there are significant differences in the reactivity score, $F(2,396)=5.59, p<0.01$; executive function score, $F(2,396)=$ $3.28, p<0.05$; and total cognitive score, $F(2,396)=5.65, p<$ 0.01 . We conducted a post hoc test using the LMATRIX syntax in SPSS, and the results verify that the performance of the DCD group was worse than that of the NC and AR groups in terms of reactivity. With regard to executive function and cognition, the DCD children scored significantly lower than the NC group's children.

TABLE 5 | Predictive effect of total movement development score on memory score.

\begin{tabular}{lcccc}
\hline & $\boldsymbol{\beta}$ & $\boldsymbol{t}$ & $\boldsymbol{\Delta} \boldsymbol{R}^{\mathbf{2}}$ & $\boldsymbol{\Delta} \boldsymbol{F}$ \\
\hline Gender & -0.09 & -1.58 & & \\
Age & $0.13^{*}$ & 2.22 & & \\
Mother's years of education & $-0.15^{*}$ & -2.16 & $0.04^{*}$ & 2.29 \\
Father's years of education & 0.04 & 0.55 & & \\
Annual household income & -0.02 & -0.43 & & \\
\hline Total movement development score & t0.20** & 3.47 & $0.04^{* *}$ & 12.06 \\
\hline${ }^{*} p<0.05$. & & & & \\
${ }^{* *} p<0.01$. & & & &
\end{tabular}

TABLE 6 | Predictive effect of total movement development score on attention score.

\begin{tabular}{lcccc}
\hline & $\boldsymbol{\beta}$ & $\boldsymbol{t}$ & $\boldsymbol{\Delta R}^{\mathbf{2}}$ & $\boldsymbol{\Delta} \boldsymbol{F}$ \\
\hline Gender & $-0.12^{\star}$ & -2.10 & & \\
Age & $-0.32^{\star \star \star}$ & -5.90 & & \\
Mother's years of education & -0.10 & -1.57 & $0.13^{\star \star \star}$ & 8.86 \\
Father's years of education & 0.06 & 0.96 & & \\
Annual household income & 0.00 & -0.08 & & \\
\hline Total movement development score & $0.14^{*}$ & 2.51 & $0.02^{*}$ & 6.31 \\
\hline${ }^{*} p<0.05$. & & & & \\
${ }^{* * *} p<0.001$. & & & &
\end{tabular}


TABLE 7 | Predictive effect of total movement development score on executive function score.

\begin{tabular}{lcccc}
\hline & $\boldsymbol{\beta}$ & $\boldsymbol{t}$ & $\boldsymbol{\Delta} \boldsymbol{R}^{\mathbf{2}}$ & $\boldsymbol{\Delta} \boldsymbol{F}$ \\
\hline Gender & 0.01 & 0.16 & & \\
Age & $-0.18^{* *}$ & -3.09 & & \\
Mother's years of education & 0.08 & 1.16 & $0.04^{*}$ & 2.30 \\
Father's years of education & -0.09 & -1.24 & & \\
Annual household income & 0.01 & 0.16 & & \\
\hline Total movement development score & 0.10 & 1.65 & 0.01 & 2.73 \\
\hline${ }^{*} p<0.05$. & & & & \\
${ }^{* *} p<0.01$. & & & &
\end{tabular}

\section{DISCUSSION}

Movement development has an observable influence on children's basic cognitive abilities. In this study, a standardized movement test (featuring 2016 Chinese norm data) and various cognitive ability tasks were used to test the movement development levels and cognitive abilities of children aged 7 to 10 years. The results suggest that there is a significant correlation between children's movement development levels and the total scores from the cognitive tests, a finding that is consistent with the relationships identified between the children's movement development levels and individual cognitive tests. Specifically, the total score for movement development is largely correlated with the scores of reaction time, executive function, memory, and attention tasks. These results are consistent with the findings and general direction of existing studies, corroborating that children's movement development may have an impact on their memory, attention, executive function, and other cognitive abilities.

The cognitive ability deviation in children with DCD manifests mainly with respect to executive function. After distinguishing between the DCD, AR, and NC group according to the norms, the results of our study's multivariate covariance analysis showed that DCD children scored significantly lower in terms of the total cognitive score compared to the other groups, which further confirmed that children with DCD generally have deviations in cognitive ability. In terms of specific cognitive tasks, this study found that, compared with the other groups, children with DCD attained significantly lower reaction time and executive function scores, which is consistent with the findings of previous studies.
The main cognitive tasks that tested the children's reaction time and executive function in this study reflected the three principal components of executive function (cognitive flexibility, inhibition control, and working memory), and the results support the conclusion of previous studies that children with DCD have difficulty with executive function $(31,34)$.

On the other hand, this study found that children in the $\mathrm{DCD}, \mathrm{AR}$, and NC groups did not show significant differences in cognitive task performance with regards to attention and memory, which differs from the conclusions of previous studies. We believe that performance deviations in respect of attention for children with DCD in existing research, occur mostly in visual tracking, due to the interference of invalid clues and other tasks $(24,25)$. Such tasks are performed through inhibition control, a principal component of executive function. Other studies have pointed out that attention performance deviation in children with DCD occurs in dual-task parallel switching (23), and attention switching - or cognitive flexibility - is also a principal component of executive function. The results in the current study, pertaining to the reaction time scores (cognitive flexibility) and executive function scores, are consistent with those of prior studies. However, regarding other cognitive tasks that examined attention only, including attention maintenance, there was no significant difference between the DCD, AR, and NC group.

Similarly, there have been some reports on performance deviation regarding memory in children with DCD that also point to the task paradigm related to children's visual perception of spatial information and the retention of motor information $(18,35,36)$ - a task paradigm that involves working memory, the third principal component of executive function. The results of executive function tasks in our study support this conclusion regarding working memory, but, when carrying out other memory tasks, there was no significant difference in the performance of children with DCD in terms of the total memory score. These findings lead us to conclude that the deviations in attention and memory in children with DCD reported in previous studies were, rather, deviations in those components of executive function associated with attention switching and working memory. The cognitive bias in children with DCD centers mainly on poor executive function rather than deficits in attention or memory.

TABLE 8 | Comparison of the scores of the DCD group, AR group, and NC group in all dimensions.

\begin{tabular}{|c|c|c|c|c|c|}
\hline Group & $\begin{array}{c}\text { Cognitive Score } \\
M(S D)\end{array}$ & $\begin{array}{l}\text { Reactivity } \\
\qquad M(S D)\end{array}$ & $\begin{array}{l}\text { Memory } \\
M(S D)\end{array}$ & $\begin{array}{l}\text { Attention } \\
M(S D)\end{array}$ & $\begin{array}{c}\text { Executive Function } \\
\qquad M(S D)\end{array}$ \\
\hline DCD group (1) & $89.09(7.31)$ & $81.17(11.61)$ & $96.98(6.59)$ & 80.45 (19.50) & 89.47 (12.31) \\
\hline NC group (3) & $92.97(8.37)$ & $86.54(11.77)$ & $98.89(8.12)$ & $86.26(20.16)$ & $93.92(12.80)$ \\
\hline$F$ value & $5.65^{\star \star}$ & $5.59^{\star \star}$ & 1.75 & 2.93 & $3.28^{*}$ \\
\hline partial Eta-Squared & 0.04 & 0.04 & 0.01 & 0.02 & 0.02 \\
\hline Post hoc test & $1<3^{\star \star}$ & $\begin{array}{l}1<2^{\star \star} \\
1<3^{\star \star}\end{array}$ & & & $1<3^{*}$ \\
\hline
\end{tabular}


Our study has selected and systematically discussed the cognitive abilities most affected by DCD as proposed by previous studies. As well as verifying the results of prior research, we inferred further that the influence of DCD on cognitive ability is mainly reflected in a child's executive function. However, due to funding and time restrictions, the present study was unable to select a larger number of more nationally representative children with DCD as samples, or to obtain a wider distribution in age/ school grade, and subsequently to discuss possible regional and age differences. Another limitation of this study was the selection of the cognitive task paradigm. In order to save on costs and time, in the children's individual tests, we chose to administer a computeraided cognitive test rather than a cognitive task paradigm based on a range of surveys in the laboratory. This created an objective restriction that prevented further discussion of the effect of DCD on cognitive ability. Finally, an additional limitation of this study concerns the cross-sectional analysis, which limited us in terms of being able to perceive the development trends. This issue will be addressed in future research.

\section{DATA AVAILABILITY STATEMENT}

The datasets generated for this study are available on request to the corresponding author.

\section{REFERENCES}

1. Gubbay SS, Ellis E, Walton JN, Court SDM. Clumsy children: a study of apraxic and agnosic defects in 21 children. Brain (1965) 88:295-312. doi: 10.1093/brain/88.2.295

2. Dare MT, Gordon N. Clumsy children: a disorder of perception and motor organisation. Dev Med Child Neurol (1970) 12:178-85. doi: 10.1111/j.14698749.1970.tb01886.x

3. Wall AE, Reid G, Paton J. The syndrome of physical awkwardness. Adv Psychol (1990) 74:283-316. doi: 10.1016/S0166-4115(08)61185-1

4. Dewey D, Wilson BN. Developmental coordination disorder: what is it? Phys Occup Ther Pediatr (2001) 20(2-3):5-27. doi: 10.1080/J006v20n02_02

5. Sigmundsson H. Perceptual deficits in clumsy children: inter- and intramodal matching approach - a window into clumsy behavior. Neural Plast (2003) 10:27-38. doi: 10.1155/NP.2003.27

6. Henderson SE, Sugden DA, Barnett AL. Movement Assessment Battery for Children. In: Examiner's Manual. 2nd ed Pearson Assessment (2007). doi: 10.1037/t55281-000

7. American Psychiatric Association. Diagnostic and Statistical Manual of Mental Disorders. 3rd ed., rev. (DSM-III-R). Washington, DC: APA (1987).

8. American Psychiatric Association. Diagnostic and Statistical Manual of Mental Disorders. 5th ed. (DSM-V). Arlington, VA: APA (2013). doi: 10.1176/appi.books. 9780890425596

9. Dewey D, Kaplan BJ, Crawford SG, Wilson BN. Developmental coordination disorder: associated problems in attention, learning, and psychosocial adjustment. Hum Mov Sci (2002) 21:905-18. doi: 10.1016/S0167-9457(02)00163-X

10. Piek JP, Dyck MJ, Francis M, Conwell A. Working memory, processing speed, and set-shifting in children with developmental coordination disorder and attention-deficit-hyperactivity disorder. Dev Med Child Neurol (2007) 49:678-83. doi: 10.1111/j.1469-8749.2007.00678.x

11. Debrabant J, Gheysen F, Caeyenberghs K, Van Waelvelde H, Vingerhoets G. Neural underpinnings of impaired predictive motor timing in children with developmental coordination disorder. Res Dev Disabil (2013) 34:1478-87. doi: 10.1016/j.ridd.2013.02.008

12. Pangelinan MM, Hatfield BD, Clark JE. Differences in movement-related cortical activation patterns underlying motor performance in children with

\section{ETHICS STATEMENT}

Approved by IRB of Beijing Normal University.

\section{AUTHOR CONTRIBUTIONS}

LK co-designed the study, collected the data, conducted most of the analyses, and wrote the first draft of the manuscript. YW co-designed the study and contributed to the writing and revising of the first draft. WD provided training for the experimenters and collected the data. YX helped in the analyses of the results and edited the language.

\section{FUNDING}

This study was supported by a grant from the Fundamental Research Funds for the Central Universities (grant number: ZYGX2015J167).

\section{ACKNOWLEDGMENTS}

We are grateful for the cooperation of the children and their parents in this study. We also thank the experimenters for their work in data collection. and without developmental coordination disorder. J Neurophysiol (2013) 109:3041-50. doi: 10.1152/jn.00532.2012

13. Licari MK, Billington J, Reid SL, Wann JP, Elliott CM, Winsor AM, et al. Cortical functioning in children with developmental coordination disorder: a motor overflow study. Exp Brain Res (2015) 233:1703-10. doi: 10.1007/s00221-015-4243-7

14. Wilson PH, Smits-Engelsman B, Caeyenberghs K, Steenbergen B, Sugden $\mathrm{D}$, Clark J, et al. Cognitive and neuroimaging findings in developmental coordination disorder: new insights from a systematic review of recent research. Dev Med Child Neurol (2017) 59:1117-29. doi: 10.1111/dmcn.13530

15. Davis CL, Tomporowski PD, Mcdowell JE, Austin BP, Miller PH, Yanasak NE, et al. Exercise improves executive function and achievement and alters brain activation in overweight children: a randomized, controlled trial. Health Psychol (2011) 30(1):91-8. doi: 10.1037/a0021766

16. Quinn JG. Towards a clarification of spatial processing. Q J Exp Psychol (1994) 47:465-80. doi: 10.1080/14640749408401120

17. Alloway TP, Archibald L. Working memory and learning in children with developmental coordination disorder and specific language impairment. J Learn Disabil (2008) 41:251-62. doi: 10.1177/0022219408315815

18. Duff SC, Logie RH. Storage and processing in visuospatial working memory. Scand J Psychol (1999) 40:251-59. doi: 10.1111/1467-9450.404124

19. Alloway TP, Temple KJ. A comparison of working memory skills and learning in children with developmental coordination disorder and moderate learning difficulties. Appl Cognit Psychol (2007) 21:473-87. doi: $10.1002 /$ acp. 1284

20. Alloway TP. Working memory, reading, and mathematical skills in children with developmental coordination disorder. J Exp Child Psychol (2007) 96:2036. doi: $10.1016 /$ j.jecp.2006.07.002

21. van Dellen T, Geuze RH. Motor response processing in clumsy children. J Child Psychol Psychiat (1988) 29:489-500. doi: 10.1111/j.1469-7610.1988. tb00739.x

22. Langaas T, Mon-Williams M, Wann JP, Pascal E, Thompson C. Eye movements, prematurity and developmental co-ordination disorder. Vision Res (1998) 38:1817-26. doi: 10.1016/S0042-6989(97)00399-4

23. Vaessen W. Clumsy children's performance on a double task. In: A. F. Kalverboer, editor. Developmental Biopsychology: Experimental Observational Studies in Children at Risk. University of Michigan Press (1990). p. 223-40. 
24. Wilson PH, Maruff P, McKenzie BE. Covert orienting of visuospatial attention in children with developmental coordination disorder. Dev Med Child Neurol (1997) 39:736-45. doi: 10.1111/j.1469-8749.1997. tb07375.x

25. Wilson PH, Maruff P. Deficits in the endogenous control of covert visuospatial attention in children with developmental coordination disorder. Hum Mov Sci (1999) 18:421-42. doi: 10.1016/S0167-9457(99)00017-2

26. Wilmut K, Brown JH, Wann JP. Attention disengagement in children with developmental coordination disorder. Disabil Rehabil (2007) 29(1):47-55. doi: 10.1080/09638280600947765

27. Fong SSM, Chung JWY, Cheng YTY, Yam TTT, Chiu HC, Fong DYT, et al. Attention during functional tasks is associated with motor performance in children with developmental coordination disorder. Medicine (2016) 95(37):e4935. doi: 10.1097/MD.0000000000004935

28. Smyth TR, Glencross DJ. Information processing deficits in clumsy children. Aust J Psychol (1986) 38:13-22. doi: 10.1080/00049538608256413

29. Smyth TR. Abnormal clumsiness in children: a defect of motor programming? Child Care Health Dev (1991) 17:283-94. doi: 10.1111/j.1365-2214.1991. tb00698.x

30. Raynor AJ. Fractionated reflex and reaction times in children with developmental coordination disorder. Motor Control (1998) 2:114-24. doi: 10.1123/mcj.2.2.114

31. Wilson PH, Ruddock S, Smits-Engelsman B, Polatajko H, Blank R. Understanding performance deficits in developmental coordination disorder: a meta-analysis of recent research. Dev Med Child Neurol (2012) 55:217-28. doi: $10.1111 /$ j.1469-8749.2012.04436.x
32. Miyake A, Friedman NP, Emerson MJ, Witzki AH, Howerter A, Wager TD. The unity and diversity of executive functions and their contributions to complex "frontal lobe" tasks: a latent variable analysis. Cognit Psychol (2000) 41:49-100. doi: 10.1006/cogp.1999.0734

33. Ke L. Developmental coordination disorders of urban children in China: Assessment, influencing factors and interventions (Unpublished doctoral dissertation). Beijing: Beijing Normal University (2019).

34. Bernardi M, Leonard HC, Hill EL, Botting N, Henry LA. Executive functions in children with developmental coordination disorder: a 2-year follow-up study. Dev Med Child Neurol (2017) 60:306-13. doi: 10.1111/dmcn.13640

35. Quinn JG, Ralston GE. Movement and attention in visual working memory. Q J Exp Psychol (1986) 38:689-703. doi: 10.1080/14640748608401621

36. Smyth MM, Pearson NA, Pendleton LR. Movement and working memory: Patterns and positions in space. Q J Exp Psychol (1988) 40:497-514. doi: $10.1080 / 02724988843000041$

Conflict of Interest Statement: The authors declare that the research was conducted in the absence of any commercial or financial relationships that could be construed as a potential conflict of interest.

Copyright (C) $2019 \mathrm{Ke}$, Duan, Xue and Wang. This is an open-access article distributed under the terms of the Creative Commons Attribution License (CC BY). The use, distribution or reproduction in other forums is permitted, provided the original author(s) and the copyright owner(s) are credited and that the original publication in this journal is cited, in accordance with accepted academic practice. No use, distribution or reproduction is permitted which does not comply with these terms. 